\title{
Bianchi Type-V Cosmological Models for Perfect Fluid with Time-Varying Gravitational and Cosmological Constant
}

\author{
Mohammed Aman Ullah, Mohammad Amjad Hossain, Mohammad Moksud Alam \\ Department of Mathematics, University of Chittagong, Chittagong, Bangladesh \\ Email: ^aman@cu.ac.bd, mah62235@gmail.com, moksud.math@cu.ac.bd
}

How to cite this paper: Ullah, M.A., Hossain, M.A. and Alam, M.M. (2017) Bianchi Type-V Cosmological Models for Perfect Fluid with Time-Varying Gravitational and Cosmological Constant. Journal of Applied Mathematics and Physics, 5, 2283-2290. https://doi.org/10.4236/jamp.2017.511185

Received: September 30, 2017 Accepted: November 26, 2017 Published: November 29, 2017

Copyright (c) 2017 by authors and Scientific Research Publishing Inc. This work is licensed under the Creative Commons Attribution International License (CC BY 4.0).

http://creativecommons.org/licenses/by/4.0/

\begin{abstract}
Einstein's field equations with variable gravitational and cosmological constants are considered in presence of perfect fluid for locally-rotationallysymmetric (LRS) Bianchi type- $\mathrm{V}$ space-time discussion in context of the particle creation. We present new shear free solutions for both absence and presence of particle creation. The solution describes the particle and entropy generation in the anisotropic cosmological models. We observe that time variation of gravitational and cosmological constant is needed for particle creation phenomena. Moreover, we obtained the particle production rate $\Gamma(t)$ for this model and discussed in detail.
\end{abstract}

\section{Keywords}

LRS Bianchi Type-V, Perfect Fluid, Gravitational Constant, Cosmological Constant, Particle Creation

\section{Introduction}

Einstein field equation in general relativity [1] and cosmology contains two parameters: Newton's gravitational constant $G$ and the cosmological constant $\Lambda$. A number of authors have considered the cosmological model with the cosmological constant $\Lambda$ as a function of cosmic time. An important role of cosmological constant with the relation $\Lambda \propto t^{-2}$ studied by Berman et al. [2] and Berman [3] [4]. The time variation of the gravitational constant $G$ was first proposed by Dirac [5] and extensively discussed in the literature by Weinberg [6] Lau [7], Lau and Prokhovnik [8] proposed generalized field equations with time-dependent $G$ and $\Lambda$, since then many authors have investigated cosmological models with variables $G$ and $\Lambda$. Arbab [9] has discussed a viscous model 
with variable $G$ and $\Lambda$ claiming that energy is conserved. Singh et al. [10] discussed a number of classes of solutions to Einstein's field equations with variable $G$ and $\Lambda$, and bulk viscosity for a flat Robertson-Walker universe in the framework of general relativity.

The Bianchi cosmologies which are spatially homogeneous and anisotropic play an important in theoretical cosmology and have been much studied since 1960s. Coley [11] has investigated Bianchi type-V spatially homogeneous imperfect fluid cosmological models which contain both viscosity and heat flow. Coley and Hoogen [12] have studied a locally-rotationally-symmetric (LRS) Bianchi type-V metric for imperfect fluid source with both viscosity and heat conduction. Singh and Beesham [13] have presented LRS Bianchi type-V cosmological models in the presence of perfect fluid with heat conduction. Singh [14] has extended the work to LRS Bianchi type- $V$ cosmological models and obtained solutions of the field equations in general relativity. A number of studies in cosmological model are performed using the gravitational constant and cosmological constant with cosmic time variable in the context of isotropic perfect fluid [9] [15] [16] and anisotropic [17] [18] [19] [20].

Recently, particle creation and in the absence of particle creation in cosmology and its phenomenological description are discussed in detail by Singh [21]. Previously, it was also attracted a lot of interests shown in [22] [23].

In this paper, we have studied the evolution and dynamics of a perfect fluid LRS Bianchi type-V models with variable $G$ and $\Lambda$ which describe the particle creation. We also try to present the exact solutions of Einstein's field equations in the case of particle creation and in the absence of particle creation.

\section{Field Equations}

We consider a LRS Bianchi type-V space time with the metric [11]

$$
\mathrm{d} s^{2}=-\mathrm{d} t^{2}+A^{2}(t) \mathrm{d} x^{2}+\mathrm{e}^{2 x} B^{2}(t)\left(\mathrm{d} y^{2}+\mathrm{d} z^{2}\right)
$$

where $A(t)$ and $B(t)$ are the cosmic scale functions. The Einstein's field equations with time-dependent $G$ and $\Lambda$ are given by Abdel-Rahaman [15].

$$
R_{i j}-\frac{1}{2} g_{i j} R=-8 \pi G(t) T_{i j}+\Lambda(t) g_{i j}
$$

where the energy-momentum tensor $T_{i j}$ is that of a perfect fluid.

For perfect fluid, $T_{i j}$ is given by

$$
T_{i j}=(p+\rho) u_{i} u_{j}+p g_{i j}
$$

where $\rho$ is the matter density, $p$ is the thermodynamics pressure and $u_{i}$ is the four-velocity vector of the fluid satisfying $u_{i} u^{i}=-1$. In a co-moving coordinate system, the field Equations (2), for the metric (1), in case of (3), read as [18]:

$$
2 \frac{\ddot{B}}{B}+\frac{\dot{B}^{2}}{B^{2}}-\frac{1}{A^{2}}=-8 \pi G(t) p+\Lambda(t),
$$




$$
\begin{aligned}
\frac{\ddot{A}}{A}+\frac{\ddot{B}}{B}+\frac{\dot{A}}{A} \frac{\dot{B}}{B}-\frac{1}{A^{2}}=-8 \pi G(t) p+\Lambda(t), \\
2 \frac{\dot{A}}{A} \frac{\dot{B}}{B}+\frac{\dot{B}^{2}}{B^{2}}-\frac{3}{A^{2}}=8 \pi G(t) \rho+\Lambda(t), \\
\frac{\dot{A}}{A}-\frac{\dot{B}}{B}=0
\end{aligned}
$$

where dot denotes the ordinary derivative with respect to the cosmic time $t$ and double dot stands for second derivative w.r. to the same.

The vanishing of the covariant divergence of the Einstein tensor leads to the following useful equation

$$
\dot{\rho}+(\rho+p)\left(\frac{\dot{A}}{A}+2 \frac{\dot{B}}{B}\right)=-\left(\frac{\dot{G}}{G} \rho+\frac{\dot{\Lambda}}{8 \pi G}\right)
$$

The average scale factor $R$ for the LRS Bianchi type- $V$ model is defined as

$$
R=\left(A B^{2}\right)^{\frac{1}{3}}
$$

The generalized mean Hubble parameter $H$ can be defined as in [24]

$$
H=\frac{\dot{a}}{a}=\frac{1}{3}\left(\frac{\dot{A}}{A}+2 \frac{\dot{B}}{B}\right)
$$

The physical quantities of observation of interest in cosmology and the expansion scalar $\theta$, the average anisotropy parameter $A_{p}$ and the shear scalar $\sigma^{2}$, are defined as [21]

$$
\begin{gathered}
\theta=u_{; i}^{i}=\left(\frac{\dot{A}}{A}+2 \frac{\dot{B}}{B}\right) \\
A_{p}=\frac{1}{3} \sum_{i=1}^{3}\left(\frac{H_{i}-H}{H}\right)^{2} \\
\sigma^{2}=\frac{1}{2} \sigma_{i j} \sigma^{i j}=\frac{1}{3}\left(\frac{\dot{A}}{A}-\frac{\dot{B}}{B}\right)^{2}
\end{gathered}
$$

The particle content of the early universe is formed from a non-interacting comoving relativistic fluid having a particle number density $n(t)$ and obeying the equation of state of the form

$$
\begin{gathered}
n=n_{0}\left(\frac{\rho}{\rho_{0}}\right)^{\frac{1}{1+\alpha}} \\
p=\alpha \rho
\end{gathered}
$$

where $n_{0} \geq 0$ and $\rho_{0} \geq 0$ are constants and $0 \leq \alpha \leq 1$. Equation (8) can be written, using Equations (14)-(15), in the form of a particle balance equation

$$
\dot{n}+3 H n=\Gamma(t) n
$$

where $\Gamma(t)$ is the particle production rate given by 


$$
\Gamma(t)=-\frac{1}{(1+\alpha) G(t)}\left[\frac{\dot{\Lambda}(t)}{8 \pi \rho(t)}+\dot{G}(t)\right]
$$

For particle creation, it is required that Equation (17) satisfies $\Gamma(t) \geq 0$. This implies that the space-time can produce matter, while the reverse case is thermodynamically forbidden. Which leads to

$$
\frac{\dot{\Lambda}(t)}{8 \pi \rho(t)}+\dot{G}(t) \leq 0
$$

The entropy $S$ generated during the particle creation is given by

$$
T \frac{\mathrm{d} S}{\mathrm{~d} t}=-\frac{1}{G(t)}\left[\frac{\dot{\Lambda}(t)}{8 \pi \rho(t)}+\dot{G}(t)\right] \rho(t) R^{3}
$$

which can be written as

$$
\frac{\mathrm{d} S}{\mathrm{~d} t}=\frac{(1+\alpha) \rho(t) R^{3}}{T} \Gamma(t)
$$

In a cosmological fluid where the density and pressure are functions of the temperature only, i.e. $\rho=\rho(T)$ and $p=p(T)$, the entropy is given by [6]

$$
S=\frac{(\rho+p) a^{3}}{T}=\frac{(1+\alpha) \rho a^{3}}{T}
$$

The total entropy of the cosmological fluid function of the particle production rate is given by

$$
S=S_{0} \mathrm{e}^{\int \Gamma(t) \mathrm{d} t}
$$

where $S_{0} \geq 0$ is a constant of integration. In the case of no particle production, i.e. $\Gamma(t)=0$, we have the usual particle conservation law of the standard cosmology, i.e.

$$
\begin{aligned}
& \dot{\rho}+3(1+\alpha) H_{p}=0 \\
& \dot{\Lambda}(t)+8 \pi \rho \dot{G}(t)=0
\end{aligned}
$$

For the case of entropy $S(t)=S_{0}=$ constant, and thus the change of entropy is zero.

\section{Solution of Field Equations}

From Equation (7), we have

$$
B=k A
$$

where $k$ is constant of integration.

From Equations (4) and (5), we have

$$
\frac{\dot{B}^{2}}{B^{2}}=\frac{\dot{A}^{2}}{A^{2}}
$$

Using the relations (25) and (26), Equations (5) and (6) yields

$$
2 \frac{\dot{A}^{2}}{A^{2}}-2 \frac{\ddot{A}}{A}-\frac{2}{A^{2}}=8 \pi G(1+\alpha) \rho
$$


Again, from the Equations (5) and (6), we have

$$
2 \Lambda+8 \pi G(t)(p-\rho)=2 \frac{\ddot{A}}{A}+4 \frac{\dot{A}^{2}}{A^{2}}-\frac{4}{A^{2}}
$$

As described in [21], we have a system of five Equations (4)-(8) with six unknowns, namely $A, B, G, \rho, \Lambda$ and $p$. To find the exact solution of the field equations, we use the power law equation. The particle creation is described due to the variation of $G$ and $\Lambda$ as shown in [18]. We obtain the solution for $\rho$, $G$ and $\Lambda$ in the following two cases:

\subsection{In the Absence of Particle Creation:}

Consider the left part of Equation (8) equal to zero i.e.

$$
\dot{\rho}+(\rho+p)\left(\frac{\dot{A}}{A}+2 \frac{\dot{B}}{B}\right)=0
$$

Using the Equations (25) and (15), the above equation can be written as

$$
\begin{aligned}
& \text { i.e. } \frac{\dot{\rho}}{\rho}+3(1+\alpha) \frac{\dot{A}}{A}=0 \\
& \text { i.e. } \rho=\frac{k_{1}}{A^{3(1+\alpha)}}
\end{aligned}
$$

where $k_{1}$ is integrating constant. Now, using Equation (29), Equation (27) reduces to

$$
G(t)=\frac{A^{3 \alpha+1}}{4 \pi(1+\alpha) k_{1}}\left\{\dot{A}^{2}-A \ddot{A}-1\right\}
$$

Again, using the Equations (25) and (26), Equations (5) + (6) give

$$
\Lambda=\frac{2}{1+\alpha} \cdot \frac{\ddot{A}}{A}+\left(\frac{3 \alpha+1}{1+\alpha}\right) \cdot \frac{\dot{A}^{2}}{A^{2}}-\left(\frac{3+\alpha}{1+\alpha}\right) \cdot \frac{1}{A^{2}}
$$

Also, from the right side of Equation (8) gives

$$
\begin{gathered}
\frac{\dot{G}}{G} \rho+\frac{\dot{\Lambda}}{8 \pi G}=0 \\
\text { i.e. } A \ddot{A}-\dot{A}^{2}+\frac{3+\alpha}{3 \alpha+1}=0 \\
\text { i.e. } A=\frac{\beta}{2 \gamma}\left(\mathrm{e}^{\gamma t}-\mathrm{e}^{-\gamma t}\right)
\end{gathered}
$$

where $\beta$ and $\gamma$ are constants. So,

$$
B=\frac{k \beta}{2 \gamma}\left(\mathrm{e}^{\gamma t}-\mathrm{e}^{-\gamma t}\right)
$$

and $R^{3}=A B^{2}$ which gives

$$
R=\frac{k \beta}{2 \gamma}\left(\mathrm{e}^{\gamma t}-\mathrm{e}^{-\gamma t}\right)
$$

The Hubble parameter and deceleration parameter are respectively given by 


$$
H=\gamma\left(\frac{1+\mathrm{e}^{-2 \gamma t}}{1-\mathrm{e}^{-2 \gamma t}}\right), \quad q=-\left(\frac{1-\mathrm{e}^{-2 \gamma t}}{1+\mathrm{e}^{-2 \gamma t}}\right)^{2}=-\tanh ^{2}(\gamma t)
$$

The energy density, gravitational and cosmological constants and expansion scalar are given by

$$
\begin{gathered}
\rho=\frac{k(2 \gamma)^{3(1+\alpha)}}{\left\{\beta\left(\mathrm{e}^{\gamma t}-\mathrm{e}^{-\gamma t}\right)\right\}^{3(1+\alpha)}} \\
G(t)=\frac{\beta^{2}-1}{4 \pi k_{1}(1+\alpha)} \cdot\left(\frac{\beta}{2 \gamma}\right)^{3 \alpha+1}\left(\mathrm{e}^{\gamma t}-\mathrm{e}^{-\gamma t}\right)^{3 \alpha+1} \\
\Lambda(t)=3 \gamma^{2}\left(\frac{\mathrm{e}^{\gamma t}+\mathrm{e}^{-\gamma t}}{\left.\mathrm{e}^{\gamma t}-\mathrm{e}^{-\gamma t}\right)^{2}-\frac{3+\alpha}{1+\alpha} \cdot \frac{4 \gamma^{2}}{\beta^{2}}\left(\mathrm{e}^{\gamma t}-\mathrm{e}^{-\gamma t}\right)^{-2}}\right. \\
\theta=3 \gamma \frac{\left(\mathrm{e}^{\gamma t}+\mathrm{e}^{-\gamma t}\right)}{\left(\mathrm{e}^{\gamma t}-\mathrm{e}^{-\gamma t}\right)}=3 \gamma \operatorname{coth}(\gamma t)
\end{gathered}
$$

The above discussion, it is clear that the Hubble's parameter, energy density, cosmological constant, deceleration parameter and expansion scalar are decreasing as functions of time. But only gravitational constant is increasing as functions of time.

Some authors [5] [16] considered that $G$ the cosmological constant is a decreasing parameter as function of time, while some other [9] [25] are taken $G$ as increasing as function of time.

\subsection{In the Case of Particle Creation}

Let us consider, $A=a t^{m}$ and $G=b t^{r}$, where $a, b, m$ and $r$ are constants.

Now, we have from Equation (27)

$$
\begin{gathered}
\rho=\frac{1}{8 \pi(1+\alpha)}\left\{\frac{2 m}{b} \cdot \frac{1}{t^{r+2}}-\frac{2}{a^{2} b} \cdot \frac{1}{t^{2 m+r}}\right\} \\
\Lambda(t)=\frac{3(1+\alpha) m^{2}-2 m}{1+\alpha} \cdot \frac{1}{t^{2}}-\frac{3 \alpha+1}{a^{2}(1+\alpha)} \cdot \frac{1}{t^{2 m}}
\end{gathered}
$$

which satisfies the Equation (8).

The particle production rate is given by (17)

$$
\Gamma(t)=\frac{1}{1+\alpha}\left[\left\{\frac{6 a^{2}(1+\alpha) m^{2} t^{2 m+1}-\left[4 m+2 m(3 \alpha+1) t^{3}\right]}{2 m a^{2} t^{2 m+2}-2 t^{4}}\right\}+\frac{r}{t}\right]
$$

The other physical quantities are

$$
\begin{gathered}
H=3 \frac{m}{t} \\
\theta=u_{; i}^{i}=3 \frac{m}{t}
\end{gathered}
$$

The physical quantities such as energy density, cosmological constant, Hub- 
ble's parameter and expansion scalar are decreasing with the increasing of the time and tend to zero when time tends to infinity. The particle creation decreasing when time increasing and tends to zero when time tends to infinity. The mass within the co-moving volume $V \propto R^{3}$ is given by $n M_{p}=\rho R^{3}$, where $n$ is the number density of the particles and $M_{p}$ the particle mass.

\section{Conclusion}

We have obtained solution for two cases: viz absence of particle creation and for particle creation of Einstein's field equation of a locally-rotationally-symmetric Bianchi type- $\mathrm{V}$ universe with cosmological constant and gravitational constant as a cosmic time. The physical quantities are realistic i.e. they behave physically for cosmological cases. The particle production rate $\Gamma(t)$ decreasing when time is increasing but when time tends to zero $\Gamma(t)$ tends to infinity which is also physical because of Big bang theory.

\section{Acknowledgements}

Authors would like to thank Professor Dr. Mohammad Ashraful Islam, Department of Mathematics, University of Chittagong, Chittagong, Bangladesh for his valuable suggestion and kind help during this work. Authors also thank the reviewers for their valuable comments and suggestions, which are really helpful in revising this paper.

\section{References}

[1] Einstein, A. $(1917,1919)$ English translation: The Principle of Relativity (Methuen, 1923, Reprinted by Dover, 1924). Sitz. Ber. Preuss. Acad. Wiss., 177 and 191.

[2] Berman, M.S., Som, M.M. and Gomide, F.M. (1989) Brans-Dicke static universes. General Relativity and Gravitation, 21, 287-292. https://doi.org/10.1007/BF00764101

[3] Berman, M.S. (1990a) Static Universe in a Modified Brans-Dicke Cosmology. International Journal of Theoretical Physics, 29, 567-570. https://doi.org/10.1007/BF00672031

[4] Berman, M.S. (1990b) Kantowski-Sachs Cosmological Models with Constant Deceleration Parameter. Nuovo Cimento B, 105, 239-242. https://doi.org/10.1007/BF02723079

[5] Dirac, P.A.M. (1937) The Cosmological Constants. Nature, 139, 323. https://doi.org/10.1038/139323a0

[6] Weinberg, S. (1989) The Cosmological Constant Problem. Reviews of Modern Physics, 61, 1-23. https://doi.org/10.1103/RevModPhys.61.1

[7] Lau, Y.K. (1985) The Large Number Hypothesis and Einstein's Theory of Gravitation. Australian Journal of Physics, 38, 547. https://doi.org/10.1071/PH850547

[8] Lau, Y.K. and Prokhovnik, S.J. (1986) The Large Numbers Hypothesis and a Relativistic Theory of Gravitation. Australian Journal of Physics, 39, 339-346. https://doi.org/10.1071/PH860339

[9] Arbab, A.I. (1997) Cosmological Models with Variable Cosmological and Gravitational "Constants" and Bulk Viscous Models. General Relativity and Gravitation, 
29, 61-74. https://doi.org/10.1023/A:1010252130608

[10] Singh, C.P., Kumar, S. and Pradhan, A. (2007) Early Viscous Universe with Variable Gravitational and Cosmological "Constants". Class. Quantum Gravity, 24, 455-474. https://doi.org/10.1088/0264-9381/24/2/011

[11] Coley, A.A. (1990) Bianchi-V Imperfect Fluid Cosmology. General Relativity and Gravitation, 22, 3-18. https://doi.org/10.1007/BF00769241

[12] Coley, A.A. and Hoogen, R.J. (1994) Qualitative Analysis of Diagonal Bianchi Type-V Imperfect Fluid Cosmological Models. Journal of Mathematical Physics, 35, 4117-4144. https://doi.org/10.1063/1.530845

[13] Singh, C.P. and Beesham, A. (2009) Locally-Rotationally-Symmetric Bianchi Type-V Cosmology with Heat Flow. Pramana-Journal of Physics, 73, 793-798. https://doi.org/10.1007/s12043-009-0147-Z

[14] Singh, C.P. (2009) Locally-Rotationally-Symmetric Bianchi Type-V Cosmology in General Relativity. Pramana-Journal of Physics, 72, 429-443. https://doi.org/10.1007/s12043-009-0038-3

[15] Abdel-Rahaman, A.M.M. (1990) A Critical Density Cosmological Model with Varying Gravitational and Cosmological “Constants”. General Relativity and Gravitation, 22, 655-663. https://doi.org/10.1007/BF00755985

[16] Beesham, A. (1986) The Cosmological Constant $(\Lambda)$ as a Possible Primordial Link to Einstein's Theory of Gravity, the Properties of Hadronic Matter and the Problem of Creation. Nuovo Cimento B, 96, 17-20. https://doi.org/10.1007/BF02725574

[17] Chakrabarty, I. and Pradhan, A. (2001) LRS Bianchi I Models with Time-Varying Gravitational and Cosmological Constants. Gravitation and Cosmology, 7, 55-57.

[18] Pradhan, A. and Yadav, V.K. (2002) Bulk Viscous Anisotropic Cosmological Models with Variable $\mathrm{G}$ and $\Lambda$. International Journal of Modern Physics D, 11, 893-912. https://doi.org/10.1142/S0218271802002050

[19] Pradhan, A., Singh, A.K. and Otarod, O. (2007) FRW Universe with Variable G and $\Lambda$-Terms. Romanian Journal of Physics, 52, 445.

[20] Ram, S., Zeyauddin, M. and Singh, C.P. (2009) Bianchi Type-V Cosmological Models with Perfect Fluid and Heat Flow in Saez-Ballester Theory. Pramana-Journal of Physics, 72, 415-427. https://doi.org/10.1007/s12043-009-0037-4

[21] Singh, C.P. (2011) Cosmological Models with Time-Varying Gravitational and Cosmological “Constants”. Astrophysics and Space Science, 331, 337-342. https://doi.org/10.1007/s10509-010-0439-2

[22] Harko, T. and Mak, M.K. (1999) Particle Creation in Cosmological Models with Varying Gravitational and Cosmological “Constants”. General Relativity and Gravitation, 31, 849-862. https://doi.org/10.1023/A:1026634204476

[23] Harko, T. and Mak, M.K. (1999) Particle Creation in Varying Speed of Light Cosmological Models. Classical and Quantum Gravity, 16, 2741. https://doi.org/10.1088/0264-9381/16/8/312

[24] Grøn, Ø. (1985) Expansion Isotropization during the Inflationary Era. Physical Review D, 32, 2522. https://doi.org/10.1103/PhysRevD.32.2522

[25] Kalligas, D., Wesson, P. and Everitt, C.W.F. (1992) Flat FRW Models with Variable $\mathrm{G}$ and $\Lambda$. General Relativity and Gravitation, 24, 351-357. https://doi.org/10.1007/BF00760411 